\title{
Vanishing influence of the band gap on the charge exchange of slow highly charged ions in freestanding single-layer $\mathrm{MoS}_{2}$
}

\author{
S. Creutzburg $\odot,{ }^{1,8, *}$ J. Schwestka $\odot,{ }^{2}$ A. Niggas $\odot,{ }^{2}$ H. Inani $\odot,{ }^{3}$ M. Tripathi,${ }^{3}$ A. George, ${ }^{4}$ R. Heller, ${ }^{1}$ R. Kozubek, ${ }^{5}$ \\ L. Madauß, ${ }^{5}$ N. McEvoy ${ }^{\circ},{ }^{6}$ S. Facsko, ${ }_{1}$ J. Kotakoski ${ }^{3},{ }^{3}$ M. Schleberger, ${ }^{5}$ A. Turchanin ${ }^{\circ},{ }^{4}$ P. L. Grande, ${ }^{7}$ \\ F. Aumayr $\left(\mathbb{0}^{2}\right.$ and R. A. Wilhelm ${ }^{1,2, \dagger}$ \\ ${ }^{1}$ Helmholtz-Zentrum Dresden-Rossendorf, Institute of Ion Beam Physics and Materials Research, \\ 01328 Dresden, Germany, European Union \\ ${ }^{2}$ TU Wien, Institute of Applied Physics, 1040 Vienna, Austria, European Union \\ ${ }^{3}$ University of Vienna, Faculty of Physics, 1090 Vienna, Austria, European Union \\ ${ }^{4}$ Friedrich Schiller University Jena, Institute of Physical Chemistry, 07743 Jena, Germany, European Union \\ ${ }^{5}$ University Duisburg-Essen, Faculty of Physics and CENIDE, 47057 Duisburg, Germany, European Union \\ ${ }^{6}$ Advanced Materials and Bioengineering Research Centre (AMBER) and School of Chemistry, Trinity College Dublin, \\ College Green, Dublin 2, Ireland, European Union \\ ${ }^{7}$ Universidade Federal do Rio Grande do Sul, Instituto de Física, Ion Implantation Laboratory, Av. Bento Gonçalves 9500, Porto Alegre, Brazil \\ ${ }^{8}$ Technische Universität Dresden, 01062 Dresden, Germany, European Union
}

(Received 21 January 2020; revised 2 June 2020; accepted 8 June 2020; published 7 July 2020)

\begin{abstract}
Charge exchange and kinetic energy loss of slow highly charged xenon ions transmitted through freestanding monolayer $\mathrm{MoS}_{2}$ are studied. Two distinct exit charge state distributions, characterized by high and low charge states, are observed. They are accompanied by smaller and larger kinetic energy losses, as well as scattering angles, respectively. High charge exchange is attributed to two-center neutralization processes, which take place in close impact collisions with the target atoms. Experimental findings are compared to graphene as a target material and simulations based on a time-dependent scattering potential model. Independent of the target material, experimentally observed charge exchange can be modeled by the same electron capture and de-excitation rates for $\mathrm{MoS}_{2}$ and graphene. A common dependence of the kinetic energy loss on the charge exchange for $\mathrm{MoS}_{2}$ as well as graphene is also observed. Considering the similarities of the zero band-gap material graphene and the $1.9 \mathrm{eV}$ band-gap material $\mathrm{MoS}_{2}$, we suggest that electron transport on the femtosecond timescale is dominated by the strong influence of the ion's Coulomb potential in contrast to the dispersion defined by the material's band structure.
\end{abstract}

DOI: 10.1103/PhysRevB.102.045408

\section{INTRODUCTION}

Material modification with ion beams represents an important technological toolkit for semiconductor doping [1], lithography mask repair [2], surface smoothing [3], and many other fields [4,5]. The level of control over the modification is determined by the mechanism of energy deposition. Besides conventional use of the ion's kinetic energy for tailoring material properties, an increased charge state of the ion also leads to substantial energy deposition [6-9]. Especially for low-dimensional materials, like nanowires [1], nanodots [10], and two-dimensional (2D) layers [11-13], ion irradiation represents a promising tool for defect engineering, which should ideally be confined to the topmost surface layer. For these classes of materials, the presence of defects can strongly affect their electronic [14], optical [15], and mechanical [16] properties.

The surface sensitivity and efficiency of defect production by slow highly charged ions (HCIs) was shown in recent

\footnotetext{
*s.creutzburg@hzdr.de

†wilhelm@iap.tuwien.ac.at
}

years for semiconducting and insulating materials [6]. A clear effect of potential energy deposition leading to structural modifications in metallic and even semimetallic surfaces (a single layer of graphene) was not observed until recently [17]. Since changes in the surfaces induced by slow highly charged ions are mediated by strong electronic excitations at the surface and subsequent lattice heating by electron-phonon coupling [18] or even Coulomb explosion [19], high electron mobility in metallic surfaces may screen the excitations rapidly and the deposited energy may dissipate before the lattice disintegrates. For materials with a band gap, surface charge and exciton screening are less efficient and the resulting energy confinement finally may lead to nanostructures such as hillocks, craters, and pits on bulk surfaces [6]. Recently, it was shown that even in suspended 2D insulating carbon nanomembranes (CNM) and in the 2D semiconductor $\mathrm{MoS}_{2}$ [7-9] nanometer-sized pores can be produced, demonstrating the surface-sensitive energy release via the rapid neutralization of a slow HCI [20]. No potential-energy-driven perforation was found in the semimetal graphene [17]. While there is a clear band-gap dependence [6] on the process leading to nanostructures using HCIs as a result of electron mobility and surface 
charge screening on the timescale of electronically mediated surface atom displacement (100 fs-10 ps), it is unclear how the initial charge transfer to an ion and subsequent potential energy deposition may depend on the band structure. In other words, is the disparity of insulators and metals with regard to HCI-induced nanostructuring a result of a different potential energy deposition mechanism on the 1 to $10 \mathrm{fs}$ timescale or different energy dissipation channels on the $100 \mathrm{fs}-10 \mathrm{ps}$ timescale?

Earlier studies of HCI-induced electron emission from metallic $\mathrm{Be}$ and insulating $\mathrm{BeO}$ surfaces as well as bulk $\mathrm{LiF}$ and $\mathrm{LiF}$ coated $\mathrm{Au}(110)$ surfaces showed that a band-gap effect is vanishing [21,22]. However, the spectroscopy of electrons gives indirect access to the phenomena, in contrast to the observation of the ions directly, which we demonstrate here.

\section{EXPERIMENTAL CONDITIONS}

We apply ion transmission spectroscopy of HCIs to freestanding $2 \mathrm{D}$ materials to probe the charge exchange dynamics in a solid surface directly in experiment. For that, measurements were performed at TU Wien using the setup as described in Ref. [23] and at the Ion Beam Center of the Helmholtz-Zentrum Dresden-Rossendorf (HZDR) [24]. Charge exchange of highly charged xenon ions through $\mathrm{MoS}_{2}$ monolayers [Fig. 1(a)] is measured as a function of the ion's kinetic energy between 10 and $260 \mathrm{keV}$ corresponding to transmission times between 2.1 and $0.5 \mathrm{fs}$, respectively, assuming an interaction length of $3.2 \AA$ (nominal $S$ - $S$ distance [25]). Highly charged xenon ions with $q=10-40$ ( $q$ indicates the ion's charge state) are produced in an electron beam ion trap $[26,27]$ commercially available from Dreebit, Germany. Ions are charge state selected by means of a Wien filter. A negative potential can be applied to the ion source (including all power supplies galvanically isolated from ground through an isolating transformer) in order to decelerate the ions down to kinetic energies of a few keV. Freestanding single-layer $\mathrm{MoS}_{2}(2 \mathrm{H}$ phase) samples are produced by chemical vapor deposition on $\mathrm{SiO}_{2}$ and transferred onto a Quantifoil supported transmission electron microscopy (TEM) grid. Various sample batches produced in Duisburg, Dublin [28], and Jena $[29,30]$, were used to compare the influence of different sample homogeneities and layer transfer methods. It should be remarked that we used $\mathrm{MoS}_{2}$ samples from three different groups with slightly varying growth conditions. Still, our results are in agreement across the set of samples. We expect that the varying degree of doping present in as-grown $\mathrm{MoS}_{2}$ due to intrinsic defects and strain will play only a minor role in our study. This becomes even more evident by the fact that doping of up to $10^{12}-10^{13}$ carriers $/ \mathrm{cm}^{2}$ in $\mathrm{MoS}_{2}$ [31] is expected, leading to a change in the band gap of less than $10 \%$ [32], which is small in relation to the band-gap difference to semimetallic graphene.

Freestanding single-layer graphene samples also on Quantifoil supported TEM grids were bought commercially from Graphenea [33]. Samples are clamped in a holder mounted on a four-axis manipulator. The pressure in the target chamber was kept below $3 \times 10^{-9}$ mbar. Samples were annealed at around $400^{\circ} \mathrm{C}$ for several hours prior to the measurements in ultra-high vacuum (UHV) in order to minimize the pres-
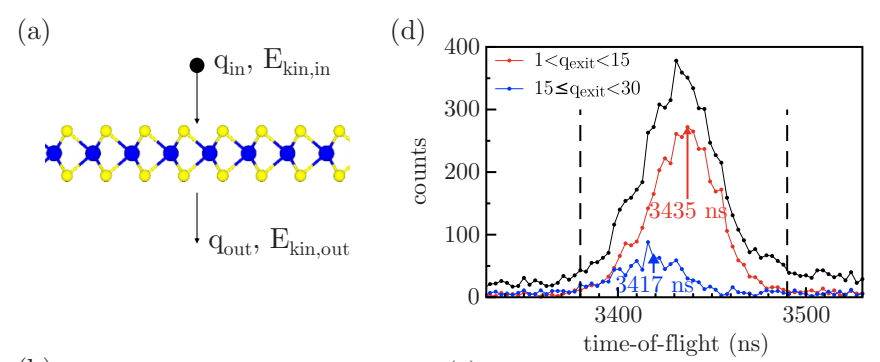

(b)

(c)
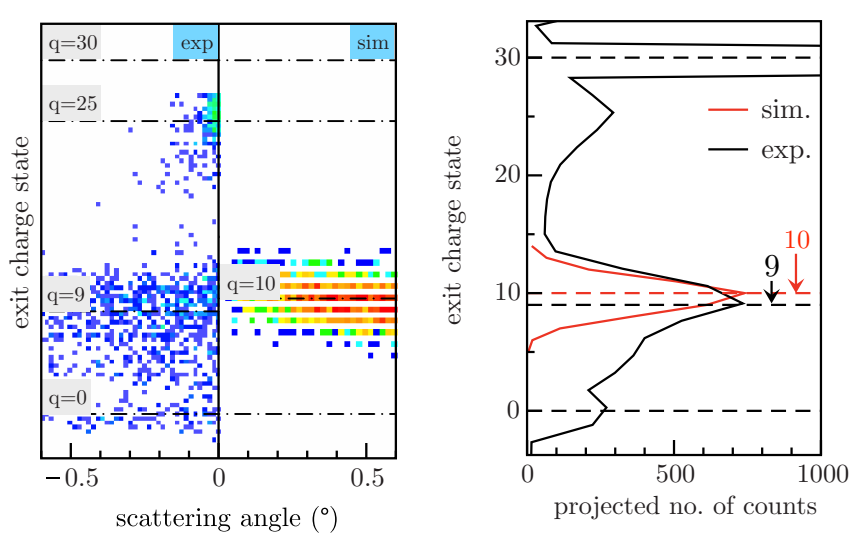

FIG. 1. (a) Sketch of ion transmission through a freestanding monolayer of $\mathrm{MoS}_{2}$. (b) Exit charge state and scattering angle resolved spectrum for incident xenon ions with a charge state of $q=30$ and kinetic energy of $87 \mathrm{keV}$ after transmission through $\mathrm{MoS}_{2}$. The experimental spectrum is filtered for transmitted ions with TOFs between 3380 and $3490 \mathrm{~ns}$, as indicated in panel (d) by dashed lines. (c) Projected number of counts integrated over scattering angles up to $0.6^{\circ}$. (d) TOF spectrum, separately filtered by exit charge states in the range of $1<q_{\text {exit }}<15$ and $15 \leqslant q_{\text {exit }}<30$, respectively.

ence of contaminations [34,35]. Some samples were also illuminated with a $6 \mathrm{~W} / 445 \mathrm{~nm}$ laser diode [36] and a spot size of $\approx 10 \mathrm{~mm}^{2}$ for a few seconds following the cleaning procedure described in Ref. [37]. Results on charge exchange for thermally annealed and laser-illuminated samples are in good agreement with one another.

At TU Wien, time of flight (TOF) of the ions was determined by measuring emitted electrons collected in a highvoltage biased surface barrier detector [38-40] and used as the start trigger. After a drift region of about $1.1 \mathrm{~m}$, ions were detected by a position-sensitive Roentdek delay line detector (DLD), whereas the impact served also as the stop trigger [23]. Furthermore, charge exchange and kinetic energy losses during the transmission through $\mathrm{MoS}_{2}$ and graphene as reference target were measured at HZDR using an electrostatic analyzer as described in Refs. [17,24,41].

\section{MODELING OF CHARGE EXCHANGE}

Charge exchange was modeled by employing the computer code TDPOT. For a comprehensive description, the reader is referred to Ref. [42]. The model describes the ion's neutralization in two steps. When the incoming ion with charge state $q_{\text {in }}$ approaches a critical distance $R_{\mathrm{C}}$ to the target surface, electrons are resonantly transferred into high-lying $n$ shells ( $n=$ principal quantum number) of the projectile according to 
the classical-over-barrier (COB) model [43]. A hollow atom with intermediate empty electron shells is formed, which is still strongly excited upon ion impact. In a second step, deexcitation of the hollow atom occurs, for which interatomic Coulombic decay (ICD) [44-51] is assumed as most prominent mechanism, as recently proposed for HCI interaction with graphene [52]. ICD is a two-center Auger de-excitation process explicitly taking into account the atomic environment of the projectile during de-excitation. Analog processes such as Penning ionization [53] and Auger de-excitation [54] were previously invoked for ion collisions, and due to the close similarity we use the term ICD here. In the model, three types of electrons are considered: the number of frozen electrons during the collision $N_{\text {core }}$, the number of electrons captured into high-lying Rydberg $n$ shells $N_{\text {capt }}(t)$, and the number of stabilized electrons $N_{\text {stab }}(t)$. The rate $\lambda(t)$ for resonant capture between the target surface and the projectile describes the time dependence of hollow atom formation. A constant capture rate $\lambda(t)=1$ a.u. is assumed for distances smaller than $R_{\mathrm{C}}$. The precise value of the capture rate is not known, but assumed to be similar to the electron orbital velocities and its exact value has only minor influence on the outcome of the model calculations. $R_{\mathrm{C}}$ is extracted from time-dependent density functional theory (TD-DFT) calculations [17]. The rate $\gamma(R)$ for hollow atom de-excitation is described by $\omega /\left(R^{8}+R_{0}^{8}\right)$, where $\omega / R_{0}^{8}=\gamma_{\max }$ indicates a maximum value at small $R$ and $R_{0}$ relates to a spatial width. A long-range limit between $R^{-6}$ and $R^{-8}$ is predicted for two-center Auger processes [55]. The parameters $R_{0}$ and $\omega$ can be estimated by a fit to literature data of decay widths for ICD active diatomic systems obtained by time-resolved pump-probe measurements or theoretical calculations [42, and references therein]. Another way to determine $R_{0}$ and $\omega$ in our model is based on a comparison with our experimental data (i.e., the exit charge-state distribution), which assumes that the measured exit charge state will be close to $q_{\text {in }}-N_{\text {stab }}(t)$. This is justified since weakly bound electrons, which still occupy outer projectile shells after transmission $N_{\text {capt }}(t \rightarrow \infty)$, will be mostly stripped by much slower autoionization processes. The parameters $R_{0}$ and $\omega$ are then determined by achieving the best agreement between $q_{\text {in }}-N_{\text {stab }}(t)$ from experiment and TDPOT. The parameters for the model obtained in this way are given in the Supplemental Material [56]. Note that our resulting parameters are also well in agreement with data in the literature on ICD rates, wherever they are available [42]. The rate parameters related to the resonant $\mathrm{COB}$ process can be varied in a reasonable range without affecting the number of stabilized electrons. This shows the sensitivity of the number of stabilized electrons mostly depending on the choice of the de-excitation rate $\gamma(R(t))$ [57]. After introducing the rates of charge transfer, the time dependence of $N_{\text {core }}, N_{\text {capt }}(t)$, and $N_{\text {stab }}(t)$ can be described by a set of coupled differential equations (1), (2), and (3):

$$
\begin{aligned}
\frac{d N_{\text {capt }}}{d t} & =\lambda(t)\left(Z-N_{\text {core }}-N_{\text {capt }}-N_{\text {stab }}\right)-\gamma(t) N_{\text {cap }}, \\
\frac{d N_{\text {stab }}}{d t} & =\gamma(t) N_{\text {cap }}, \\
\frac{d N_{\text {core }}}{d t} & =0
\end{aligned}
$$

where $Z$ is the nuclear charge of the projectile.

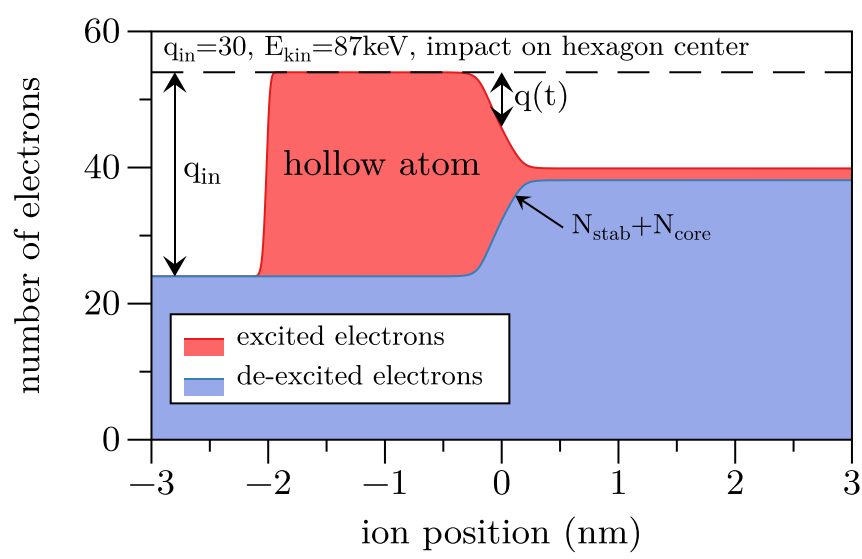

FIG. 2. Description of the charge exchange model. Number of electrons contributing to hollow atom formation and subsequent de-excitation for different positions along the ion's trajectory. Initial conditions of $q_{\text {in }}=30$ and $E_{\mathrm{kin}}=87 \mathrm{keV}$ are chosen for transmission through the middle of the hexagon formed by the carbon atoms. During neutralization, the ion stabilizes 14 electrons.

In order to describe the kinetic energy loss, the solutions of (1)-(3) are used in a description for the interaction potential between ion and target atoms. We use an approach explained in Refs. [42,58], where the partially dressed projectile is split into an unscreened part of charge state $q(t)=q_{\text {in }}-N_{\text {capt }}(t)-$ $N_{\text {stab }}(t)$ and a screened part of charge state $Z-q(t)$. Since a comprehensive description is given in Refs. [42,58], further details are omitted here. In order to consider simultaneous scattering on more than one target atom, we sum over all two-body potentials for all target atoms in the simulation cell. As it turns out, the potential vanishes quickly with interatomic separation, such that only nearest and next-nearest neighbors contribute significantly to the interaction.

The screening lengths are modified by a scaling parameter accounting for the strongly excited interaction system as described in Ref. [42]. We want to emphasize that our model can describe the charge-state distribution after transmission solely by taking the material structure and the ICD rate into account. To resemble the condition in the ion transmission experiment, the initial ion impact positions were sampled across a unit cell in the simulation. The cell is spanned by the primitive vectors $\vec{a}_{1}=a(\sqrt{3} / 2,1 / 2)$ and $\vec{a}_{2}=a(\sqrt{3} / 2,-1 / 2)$ with $a_{\text {graphene }}=$ $0.246 \mathrm{~nm}$ [59] and $a_{\mathrm{MoS}_{2}}=0.316 \mathrm{~nm}$ [25], respectively. The initial $z$ position of the ion is chosen to be $>R_{\mathrm{C}}$. Trajectories are then calculated by solving Newton's equations of motion using the Runge-Kutta method. A visualization of the solutions of Eqs. (1)-(3) as a function of the ion's position is shown in Fig. 2 for ion transmission of $\mathrm{Xe}^{30+}$ and $E_{\text {kin }}=$ $87 \mathrm{keV}$ through the middle of a carbon hexagon in graphene.

\section{RESULTS}

An experimentally obtained charge exchange spectrum for incident $\mathrm{Xe}^{30+}$ ions transmitted through $\mathrm{MoS}_{2}$ with an initial kinetic energy of $87 \mathrm{keV}$ is shown in Fig. 1(b) on the left. To minimize noise in the spectrum, only scattering events are shown for ions, which possess TOFs between 3380 and 3490 ns, as indicated in Fig. 1(d) by dashed lines. In Fig. 1(b), two distributions are visible at high charge states $\geqslant 15$ and at 


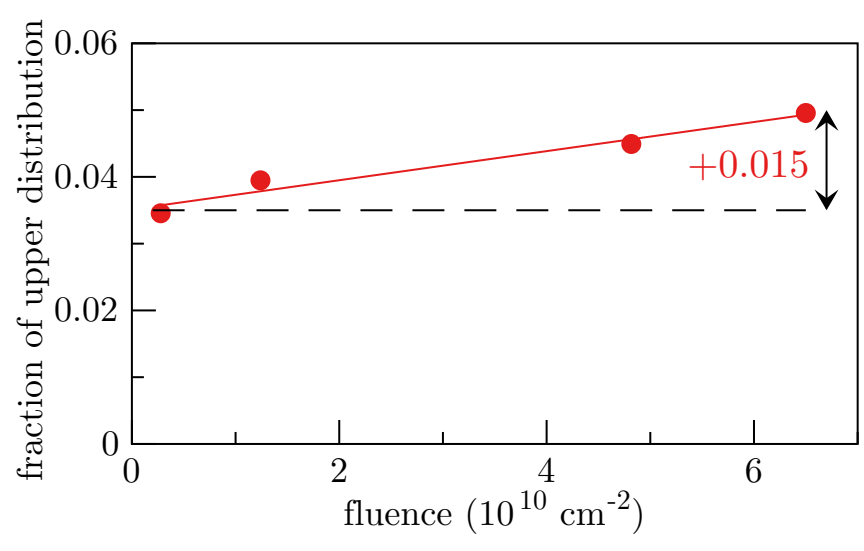

FIG. 3. Fraction of the upper charge state distribution normalized to the total number of counts as a function of applied fluence.

low charge states $<15$, respectively. The distribution located at high charge states with its projected mean value at around $q=25$ is limited to scattering angles below $0.2^{\circ}$. This implies that the corresponding scattering events take place at large impact parameters between the incident ions and the target atoms. One sample was irradiated stepwise with highly charged xenon ions with charge states between 25 and 40 at a kinetic energy of $87 \mathrm{keV}$. After each irradiation step, charge exchange spectra were recorded for the conditions applied in Fig. 1. The fraction of the upper charge state distribution normalized to the total number of scattered ions is shown in Fig. 3. An increase of the normalized intensity of the upper charge state distribution with the applied fluence by $1.5 \times 10^{-2}$ is observed. During the irradiations, an area fraction of $5 \times 10^{-3}$ is damaged by HCI induced nanopore formation as estimated from Refs. [9,60]. Because of the similar values (within a factor of 3 here), we attribute the occurrence of the upper charge state distribution to the transmission through nanopores and defective areas, which are present from the beginning on and increase in density with the applied fluence. Indeed, TEM images of pristine, freestanding $\mathrm{MoS}_{2}$ samples already reveal a non-negligible contribution of defective areas [9,29].

The lower charge state distribution presented in Fig. 1(b) is centered at a mean charge state of $q=9$ and also contains scattering angles larger than $0.2^{\circ}$. Therefore, these ions stem from collisions occurring at smaller impact parameters. The mean value of their TOFs is $3435 \mathrm{~ns}$. By taking the mean TOF of the upper distribution (3417 ns) as a reference, kinetic energy losses in the order of $1000 \mathrm{eV}$ for the lower distribution are estimated. The simulated spectrum is added in Fig. 1(b) on the right with a projected mean exit charge state of 10 , in fair agreement with the experimental spectrum.

The dependence of the mean number of captured electrons on the ion's inverse velocity (proportional to the transmission time) is shown in Fig. 4(a) for $\mathrm{MoS}_{2}$. Data from experiments and simulations are shown together. Additionally, data for graphene are presented in Fig. 4(b) for comparison [61]. Good agreement is observed between the experimental and simulated data. Note that charge exchange for both target materials was modeled by a common de-excitation function $\gamma(R)$. The rate at which electrons are quenched into states with higher

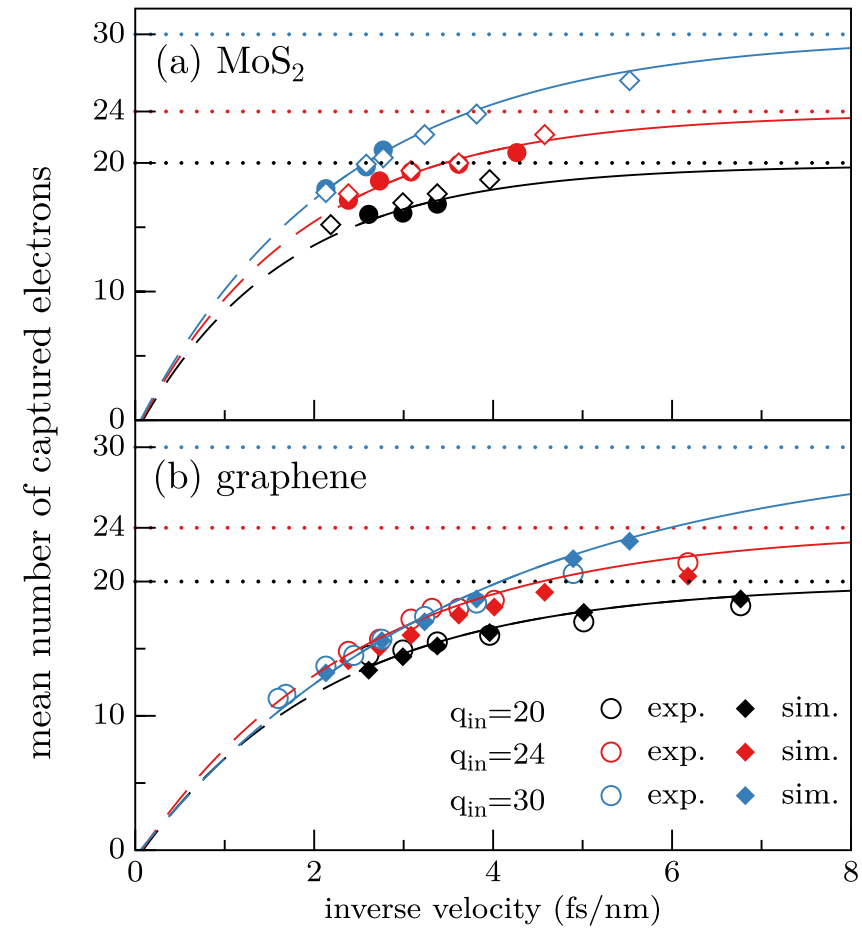

FIG. 4. Dependence of the mean number of captured electrons on the ion's inverse velocity for xenon ions transmitted through $\mathrm{MoS}_{2}$ (a) and graphene (b) for incident ions of charge states 20, 24, and 30. Solid lines are fits to the data according to an exponential decay of the charge state (see text). The fits are extrapolated to zero inverse velocity as dashed lines. The maximum experimental uncertainty of the mean number of captured electrons is estimated by \pm 1 and originates mainly from a FWHM of 3-4 for the distributions.

binding energy due to ICD is the most sensitive unknown parameter in the TDPOT model. The best agreement with our experimental data of the exit charge state distribution is found if a dependence of the de-excitation rate on the incident charge state is assumed. This $q$-dependent factor decreases by $(35 \pm 5) \%$ with increasing incident charge state from 20 to 30. A charge state dependence of ICD has not been discussed in the literature so far, because ICD is typically invoked for cluster systems where one atom carries a single core hole. However, a charge state dependence can be expected in our case, since the intermediate hollow atom state of the projectile depends on the initial charge state; i.e., the occupation of atomic states in the projectile is different depending on the details of the first electron transport processes (COB transport). The hollow atom, as a precursor for ICD, still carries information on the incident charge state.

In order to investigate the timescale dynamics of neutralization, it is assumed that neutralization follows an exponential time dependence according to $N_{\text {stab,exp }}(v)=$ $\left(q_{\text {in }}-q_{\text {eq }}\right)\left(1-\exp \left(-\frac{v_{\mathrm{N}}}{v}\right)\right)[62,63] . q_{\text {in }}$ is the charge state of the incident ions with velocity $v . v_{\mathrm{N}}$ denotes the neutralization parameter, i.e., the (inverse) charge state decay constant. $q_{\text {eq }}$ is the equilibrium charge state given by $q_{\mathrm{eq}}=Z^{1 / 3} v / v_{0}$ [64], which represents the average charge state of an ion with nuclear charge $Z$ moving through a solid with velocity $v\left(v_{0}\right.$ is the Bohr velocity). Neutralization parameters determined 
TABLE I. Neutralization parameters from experiments and extracted neutralization times.

\begin{tabular}{lccccc}
\hline \hline & \multicolumn{2}{c}{$v_{\mathrm{N}}[\mathrm{nm} / \mathrm{fs}]$} & & \multicolumn{2}{c}{$t_{\mathrm{N}}[\mathrm{fs}]$} \\
\cline { 2 - 3 } \cline { 5 - 6 } Qin & $\mathrm{MoS}_{2}$ & Graphene & & $\mathrm{MoS}_{2}$ & Graphene \\
\hline 20 & $0.62(7)$ & $0.47(4)$ & & $2.0(2)$ & $1.9(2)$ \\
24 & $0.55(5)$ & $0.41(3)$ & & $2.3(2)$ & $2.4(2)$ \\
30 & $0.44(3)$ & $0.28(2)$ & & $3.1(2)$ & $3.8(3)$ \\
\hline \hline
\end{tabular}

from the experimental data are shown in Table I for $\mathrm{MoS}_{2}$ and graphene.

Neutralization times can be estimated by calculating the ratio $L_{\mathrm{N}} / v_{\mathrm{N}}$, where $L_{\mathrm{N}}$ represents the neutralization length. For graphene, the neutralization length is approximated by the critical distance of the first electron transfer $R_{\mathrm{C}}$ obtained by TD-DFT simulations [17], which provided values between 9 and $11 \AA$ depending on the incident charge state. In the case of $\mathrm{MoS}_{2}$, additionally the thickness of the triple-layer structure (nominal S-S distance of $3.2 \AA$ [25]) was taken into account. Neutralization times are added to Table I. A fair agreement in the neutralization times for the same charge state of the incident ions is observed. Hence, the differences in the neutralization for both target materials is explained essentially by the different thicknesses. This is supported by the fact that the velocity dependence of the number of captured electrons can be modeled by the same de-excitation rates for both materials.

Additionally, experimental data for $\mathrm{Xe}^{30+}$ interaction with $\mathrm{C}_{60}$ (fullerene) are available in the literature [65], where a neutralization parameter of $0.35 \mathrm{~nm} / \mathrm{fs}$ is reported. We find a neutralization parameter of $0.28 \mathrm{~nm} / \mathrm{fs}$ for graphene from our experiments, which corresponds well to results in Ref. [65].

In addition to charge exchange, the charge-state-dependent kinetic energy loss for graphene and $\mathrm{MoS}_{2}$ was measured using an electrostatic analyzer. The dependence of the kinetic energy loss on the exit charge state is shown in Fig. 5(a) for $\mathrm{MoS}_{2}$ and in Fig. 5(b) for graphene. Ions with incident charge states of $q_{\mathrm{in}}=20$ and 28 and kinetic energies of $E_{\text {kin }}=75$ and $82 \mathrm{keV}$, respectively, were chosen. An increase of the energy loss toward lower exit charge states and higher incident charge states is observed for both target materials. A dependence of the kinetic energy loss on the exit charge state was already reported for CNMs [41] and graphene [17]. The kinetic energy losses obtained from TDPOT simulations are added to Figs. 5(a) and 5(b). A fair agreement between the simulated and measured values can be seen. However, the slope of the exit charge state dependence is shallower for the simulated values compared to the measured ones in case of $\mathrm{MoS}_{2}$. Taking additionally the consistency in the velocity dependence of the number of captured electrons shown in Fig. 4 into account, an agreement of simulations with experimental data is found in charge exchange, scattering angles, and energy loss, simultaneously.

\section{DISCUSSION AND CONCLUSIONS}

The similar neutralization behavior of graphene and $\mathrm{MoS}_{2}$ indicates a common nature of a two-center de-excitation

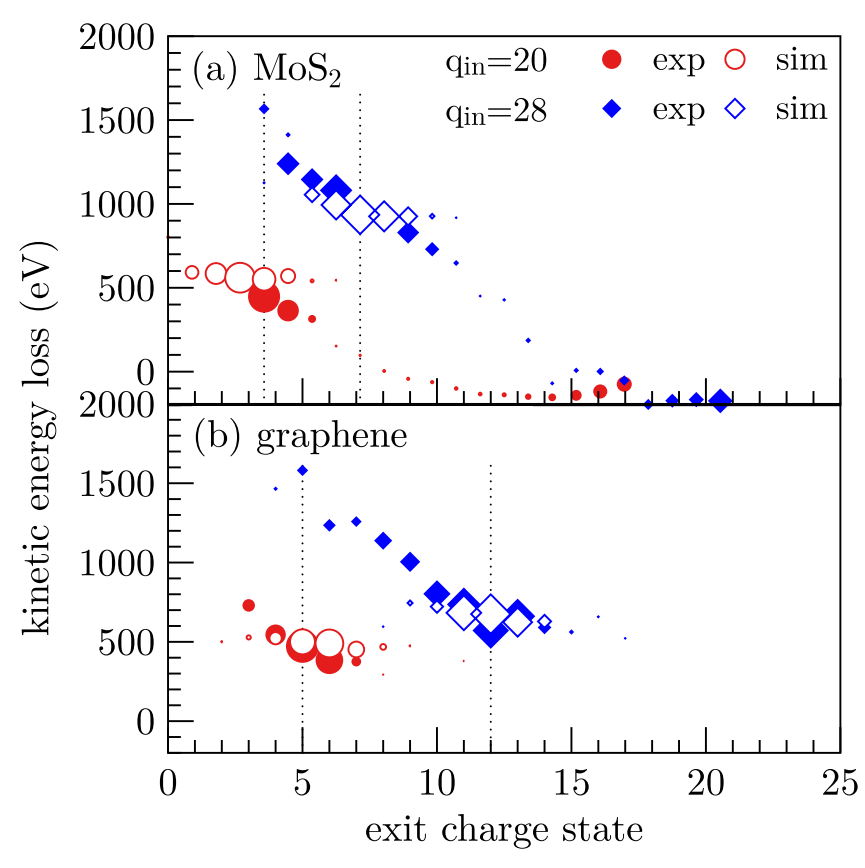

FIG. 5. Kinetic energy loss vs exit charge state for xenon ions transmitted through $\mathrm{MoS}_{2}$ (a) and graphene (b). Incident ions with charge state and kinetic energy of $q_{\text {in }}=20, E_{\text {kin }}=75 \mathrm{keV}$ and $q_{\text {in }}=$ $28, E_{\mathrm{kin}}=82 \mathrm{keV}$, respectively, were used. The size of the symbols indicates the ion abundance.

process for both target materials. ICD resembles an Auger process, in which the potential energy of an excited electron is transferred as kinetic energy to an electron of a target atom in close proximity, causing the emission of a target electron itself. Thus, the rates depend on the density of the electrons, which take part in the process. Assuming that only valence electrons with ionization energies of $\lesssim 10 \mathrm{eV}$ contribute to ICD, only one electron per target atom (C, Mo, or S) may be emitted. Note that in a subsequent ionization, the second valence electron has a higher ionization potential and thus its contribution to ICD will be lower. Consequently, the electron density corresponds to the atomic density, for which values of $0.38 \AA^{-2}$ [59] and $0.34 \AA^{-2}$ [25] in cases of graphene and $\mathrm{MoS}_{2}$, respectively, are determined. Because of the similar densities, ICD rates in the same order of magnitude can be expected.

Previously, we could exclude the contribution of other neutralization processes to the hollow atom decay for HCI interaction with graphene [52]. Since $\mathrm{Xe}-\mathrm{C}$ represents a very asymmetric target-projectile system, especially side-feeding processes could be ruled out $[66,67]$. Here, we cannot use the same argument to exclude side-feeding processes as a competing de-excitation channel for the heavier target elements (Mo and S). The occurrence of side-feeding processes between the inner target atom's and projectile's shells depends on the distance of minimal approach. At higher kinetic energies in the $\mathrm{MeV}$ range, projectile and target approach closer, rendering side-feeding processes more efficient [68,69]. Still, molybdenum $L$-shell x-ray emission seems to be suppressed for kinetic energies below $350 \mathrm{keV}$ of highly charged xenon ions [70-72] impinging on a 
molybdenum target, suggesting a reduced side-feeding efficiency. In the present work, kinetic energies well below $350 \mathrm{keV}$ are used and corresponding distances of minimal approach are $>0.3 \AA$, reducing the probability of side-feeding significantly.

To summarize, slow HCI transmission through freestanding single layers of semimetallic graphene and semiconducting $\mathrm{MoS}_{2}$ shows similar neutralization dynamics and kinetic energy-loss mechanisms. In comparison with a model taking two-center Auger-type de-excitation based on ICD into account, we see only minor differences between these two materials with regard to ion neutralization and potential energy deposition (cf. Table I). The majority of the ion's energy, i.e., the potential energy, is transferred to both targets very similarly. We conclude that differences in nanostructuring efficiencies of graphene and $\mathrm{MoS}_{2}$ are the result of electronic excitation dynamics in the surface after the ion has long passed.

\section{ACKNOWLEDGMENTS}

We gratefully acknowledge the help from M. Steinert and from the colleagues of the Ion Beam Center at HZDR for technical support. We wish to thank U. Lucchesi for her assistance during the Raman measurements (see the Supplemental Material [56]). The authors thank the Deutsche Forschungsgemeinschaft (DFG) for financial support WI 4691/1-1 Project No. 322051344, SCHL 384/20-1 Project No. 406129719, Project C5 within the SFB 1242 "Non-equilibrium Dynamics of Condensed Matter in the Time Domain" Project No. 278162697, TU149/9-1 Project No. 397373225, SFB 1375 "NOA" Project B2 Project No. 398816777, and the Thüringer Ministerium für Wirtschaft, Wissenschaft und Digitale Gesellschaft via FGR 0088 2D-Sens. Financial support from the Austrian Science Fund (FWF) (Projects No. Y1174-N36, No. P31605-N36, and No. I3181-N36) is acknowledged. J.S. gratefully acknowledges financial support from the TU Wien doctoral collage TU-D.
[1] C. Ronning, C. Borschel, S. Geburt, and R. Niepelt, Ion beam doping of semiconductor nanowires, Mater. Sci. Engin. R 70, 30 (2010).

[2] T. Amano, Y. Nishiyama, H. Shigemura, T. Terasawa, O. Suga, K. Shiina, F. Aramaki, A. Yasaka, T. Abe, and H. Mohri, FIB mask repair technology for EUV mask, Proc. SPIE 7379, 73792L (2009).

[3] F. Frost, B. Ziberi, A. Schindler, and B. Rauschenbach, Surface engineering with ion beams: From self-organized nanostructures to ultra-smooth surfaces, Appl. Phys. A 91, 551 (2008).

[4] G. Rizza, P. E. Coulon, V. Khomenkov, C. Dufour, I. Monnet, M. Toulemonde, S. Perruchas, T. Gacoin, D. Mailly, X. Lafosse, C. Ulysse, and E. A. Dawi, Rational description of the ion-beam shaping mechanism, Phys. Rev. B 86, 035450 (2012).

[5] F. Chen, Micro- and submicrometric waveguiding structures in optical crystals produced by ion beams for photonic applications, Laser Photon. Rev. 6, 622 (2012).

[6] F. Aumayr, S. Facsko, A. S. El-Said, C. Trautmann, and M. Schleberger, Single ion induced surface nanostructures: A comparison between slow highly charged and swift heavy ions, J. Phys.: Condens. Matter 23, 393001 (2011).

[7] R. Ritter, R. A. Wilhelm, M. Stöger-Pollach, R. Heller, A. Mücklich, U. Werner, H. Vieker, A. Beyer, S. Facsko, A. Gölzhäuser, and F. Aumayr, Fabrication of nanopores in 1-nmthick carbon nanomembranes with slow highly charged ions, Appl. Phys. Lett. 102, 063112 (2013).

[8] R. A. Wilhelm, E. Gruber, R. Ritter, R. Heller, A. Beyer, A. Turchanin, N. Klingner, R. Hübner, M. Stöger-Pollach, H. Vieker, G. Hlawacek, A. Gölzhäuser, S. Facsko, and F. Aumayr, Threshold and efficiency for perforation of 1-nm-thick carbon nanomembranes with slow highly charged ions, 2D Mater. 2, 035009 (2015).

[9] R. Kozubek, M. Tripathi, M. Ghorbani-Asl, S. Kretschmer, L. Madauß, E. Pollmann, M. O’Brien, N. McEvoy, U. Ludacka, T. Susi, G. S. Duesberg, R. A. Wilhelm, A. V. Krasheninnikov, J. Kotakoski, and M. Schleberger, Perforating freestanding molybdenum disulfide monolayers with highly charged ions, J. Phys. Chem. Lett. 10, 904 (2019).
[10] H. Holland-Moritz, A. Ilinov, F. Djurabekova, K. Nordlund, and C. Ronning, Sputtering and redeposition of ion-irradiated $\mathrm{Au}$ nanoparticle arrays: Direct comparison of simulations to experiments, New J. Phys. 19, 013023 (2017).

[11] M. Ghorbani-Asl, S. Kretschmer, D. E. Spearot, and A. V. Krasheninnikov, Two-dimensional $\mathrm{MoS}_{2}$ under ion irradiation: From controlled defect production to electronic structure engineering, 2D Mater. 4, 025078 (2017).

[12] Z. Li and F. Chen, Ion beam modification of two-dimensional materials: Characterization, properties, and applications, Appl. Phys. Rev. 4, 011103 (2017).

[13] M. Schleberger and J. Kotakoski, 2D material science: Defect engineering by particle irradiation, Materials 11, 1885 (2018).

[14] K. F. Mak and J. Shan, Photonics and optoelectronics of 2D semiconductor transition metal dichalcogenides, Nat. Photon. 10, 216 (2016).

[15] Z. Sun, A. Martinez, and F. Wang, Optical modulators with 2D layered materials, Nat. Photon. 10, 227 (2016).

[16] D. Akinwande, C. J. Brennan, J. S. Bunch, P. Egberts, J. R. Felts, H. Gao, R. Huang, J.-S. Kim, T. Li, Y. Li, K. M. Liechti, N. Lu, H. S. Park, E. J. Reed, P. Wang, B. I. Yakobson, T. Zhang, Y.-W. Zhang, Y. Zhou, and Y. Zhu, A review on mechanics and mechanical properties of 2D materials? Graphene and beyond, Extreme Mech. Lett. 13, 42 (2017).

[17] E. Gruber, R. A. Wilhelm, R. Pétuya, V. Smejkal, R. Kozubek, A. Hierzenberger, B. C. Bayer, I. Aldazabal, A. K. Kazansky, F. Libisch, A. V. Krasheninnikov, M. Schleberger, S. Facsko, A. G. Borisov, A. Arnau, and F. Aumayr, Ultrafast electronic response of graphene to a strong and localized electric field, Nat. Commun. 7, 13948 (2016).

[18] A. S. El-Said, R. Heller, W. Meissl, R. Ritter, S. Facsko, C. Lemell, B. Solleder, I. C. Gebeshuber, G. Betz, M. Toulemonde, W. Möller, J. Burgdörfer, and F. Aumayr, Creation of Nanohillocks on $\mathrm{CaF}_{2}$ Surfaces by Single Slow Highly Charged Ions, Phys. Rev. Lett. 100, 237601 (2008).

[19] S. Facsko, R. Heller, A. S. El-Said, W. Meissl, and F. Aumayr, Surface nanostructures by single highly charged ions, J. Phys.: Condens. Matter 21, 224012 (2009). 
[20] R. M. Papaléo, M. R. Silva, R. Leal, P. L. Grande, M. Roth, B. Schattat, and G. Schiwietz, Direct Evidence for Projectile Charge-State Dependent Crater Formation Due to Fast Ions, Phys. Rev. Lett. 101, 167601 (2008).

[21] G. Schiwietz, K. Czerski, M. Roth, P. L. Grande, V. Koteski, and F. Staufenbiel, Evidence for an Ultrafast Breakdown of the BeO Band Structure Due to Swift Argon and Xenon Ions, Phys. Rev. Lett. 105, 187603 (2010).

[22] H. Khemliche, T. Schlathölter, R. Hoekstra, R. Morgenstern, and S. Schippers, Hollow Atom Dynamics on LiF Covered $\mathrm{Au}(111)$ : Role of the Surface Electronic Structure, Phys. Rev. Lett. 81, 1219 (1998).

[23] J. Schwestka, D. Melinc, R. Heller, A. Niggas, L. Leonhartsberger, H. Winter, S. Facsko, F. Aumayr, and R. A. Wilhelm, A versatile ion beam spectrometer for studies of ion interaction with 2D materials, Rev. Sci. Instrum. 89, 085101 (2018).

[24] R. A. Wilhelm, E. Gruber, V. Smejkal, S. Facsko, and F. Aumayr, Charge-state-dependent energy loss of slow ions. I. Experimental results on the transmission of highly charged ions, Phys. Rev. A 93, 052708 (2016).

[25] J. A. Wilson and A. D. Yoffe, The transition metal dichalcogenides discussion and interpretation of the observed optical, electrical and structural properties, Adv. Phys. 18, 193 (1969).

[26] M. Schmidt, H. Peng, G. Zschornack, and S. Sykora, A compact electron beam ion source with integrated Wien filter providing mass and charge state separated beams of highly charged ions, Rev. Sci. Instrum. 80, 063301 (2009).

[27] G. Zschornack, M. Kreller, V. P. Ovsyannikov, F. Grossman, U. Kentsch, M. Schmidt, F. Ullmann, and R. Heller, Compact electron beam ion sources/traps: Review and prospects, Rev. Sci. Instrum. 79, 02 A703 (2008).

[28] M. O’Brien, N. McEvoy, T. Hallam, H. Y. Kim, N. C. Berner, D. Hanlon, K. Lee, J. N. Coleman, and G. S. Duesberg, Transition metal dichalcogenide growth via close proximity precursor supply, Sci. Rep. 4, 7374 (2014).

[29] S. Shree, A. George, T. Lehnert, C. Neumann, M. Benelajla, C. Robert, X. Marie, K. Watanabe, T. Taniguchi, U. Kaiser, B. Urbaszek, and A. Turchanin, High optical quality of $\mathrm{MoS}_{2}$ monolayers grown by chemical vapor deposition, 2D Mater. 7, 015011 (2019).

[30] A. George, C. Neumann, D. Kaiser, R. Mupparapu, T. Lehnert, U. Hübner, Z. Tang, A. Winter, U. Kaiser, I. Staude, and A. Turchanin, Controlled growth of transition metal dichalcogenide monolayers using Knudsen-type effusion cells for the precursors, J. Phys. Mater. 2, 016001 (2019).

[31] W. H. Chae, J. D. Cain, E. D. Hanson, A. A. Murthy, and V. P. Dravid, Substrate-induced strain and charge doping in CVDgrown monolayer $\mathrm{MoS}_{2}$, Appl. Phys. Lett. 111, 143106 (2017).

[32] L.-J. Kong, G.-H. Liu, and L. Qiang, Electronic and optical properties of O-doped monoalayer $\mathrm{MoS}_{2}$, Comput. Mater. Sci. 111, 416 (2016).

[33] https://www.graphenea.com/.

[34] W. Xie, L. T. Weng, K. M. Ng, C. K. Chan, and C. M. Chan, Clean graphene surface through high temperature annealing, Carbon 94, 740 (2015).

[35] W. Chen, J. Zhao, J. Zhang, L. Gu, Z. Yang, X. Li, H. Yu, X. Zhu, R. Yang, D. Shi, X. Lin, J. Guo, X. Bai, and G. Zhang, Oxygen-assisted chemical vapor deposition growth of large single-crystal and high-quality monolayer $\mathrm{MoS}_{2}$, J. Am. Chem. Soc. 137, 15632 (2015).

[36] https://www.lasertack.com/.

[37] M. Tripathi, A. Mittelberger, K. Mustonen, C. Mangler, J. Kotakoski, J. C. Meyer, and T. Susi, Cleaning graphene: Comparing heat treatments in air and in vacuum, Phys. Status Solidi RRL 11, 1700124 (2017).

[38] G. Lakits, F. Aumayr, and H. P. Winter, Statistics of ion-induced electron emission from a clean metal surface, Rev. Sci. Instrum. 60, 3151 (1989).

[39] F. Aumayr, G. Lakits, and H. P. Winter, On the measurement of statistics for particle-induced electron-emission from a clean metal surface, Appl. Surface Sci. 47, 139 (1991).

[40] H. Kurz, F. Aumayr, C. Lemell, K. Töglhofer, and H. P. Winter, Neutralization of slow multicharged ions at a clean gold surface: Electron-emission statistics, Phys. Rev. A 48, 2192 (1993).

[41] R. A. Wilhelm, E. Gruber, R. Ritter, R. Heller, S. Facsko, and F. Aumayr, Charge Exchange and Energy Loss of Slow Highly Charged Ions in 1-nm-thick Carbon Nanomembranes, Phys. Rev. Lett. 112, 153201 (2014).

[42] R. A. Wilhelm and P. L. Grande, Unraveling energy loss processes of low energy heavy ions in 2D materials, Commun. Phys. 2, 89 (2019).

[43] J. Burgdörfer, P. Lerner, and F. W. Meyer, Above-surface neutralization of highly charged ions: The classical over-the-barrier model, Phys. Rev. A 44, 5674 (1991).

[44] L. S. Cederbaum, J. Zobeley, and F. Tarantelli, Giant Intermolecular Decay and Fragmentation of Clusters, Phys. Rev. Lett. 79, 4778 (1997).

[45] S. Marburger, O. Kugeler, U. Hergenhahn, and T. Möller, Experimental Evidence for Interatomic Coulombic Decay in $\mathrm{Ne}$ Clusters, Phys. Rev. Lett. 90, 203401 (2003).

[46] T. Jahnke, A. Czasch, M. S. Schöffler, S. Schössler, A. Knapp, M. Käsz, J. Titze, C. Wimmer, K. Kreidi, R. E. Grisenti, A. Staudte, O. Jagutzki, U. Hergenhahn, H. Schmidt-Böcking, and R. Dörner, Experimental Observation of Interatomic Coulombic Decay in Neon Dimers, Phys. Rev. Lett. 93, 163401 (2004).

[47] W. Iskandar, J. Matsumoto, A. Leredde, X. Fléchard, B. Gervais, S. Guillous, D. Hennecart, A. Méry, J. Rangama, C. L. Zhou, H. Shiromaru, and A. Cassimi, Interatomic Coulombic Decay as a New Source of Low Energy Electrons in Slow Ion-Dimer Collisions, Phys. Rev. Lett. 114, 033201 (2015).

[48] R. Santra, J. Zobeley, and L. S. Cederbaum, Electronic decay of valence holes in clusters and condensed matter, Phys. Rev. B 64, 245104 (2001).

[49] T. Miteva, S. Kazandjian, P. Kolorenč, P. Votavová, and N. Sisourat, Interatomic Coulombic Decay Mediated by Ultrafast Superexchange Energy Transfer, Phys. Rev. Lett. 119, 083403 (2017).

[50] J. L. Hemmerich, R. Bennett, and S. Y. Buhmann, The influence of retardation and dielectric environments on interatomic Coulombic decay, Nat. Commun. 9, 2934 (2018).

[51] R. Bennett, P. Votavová, P. Kolorenč, T. Miteva, N. Sisourat, and S. Y. Buhmann, Virtual Photon Approximation for Three-Body Interatomic Coulombic Decay, Phys. Rev. Lett. 122, 153401 (2019).

[52] R. A. Wilhelm, E. Gruber, J. Schwestka, R. Kozubek, T. I. Madeira, J. P. Marques, J. Kobus, A. V. Krasheninnikov, M. Schleberger, and F. Aumayr, Interatomic Coulombic Decay: 
The Mechanism for Rapid Deexcitation of Hollow Atoms, Phys. Rev. Lett. 119, 103401 (2017).

[53] F. M. Penning, Über Ionisation durch metastabile Atome, Naturwissensch. 15, 818 (1927).

[54] H. D. Hagstrum, Theory of auger ejection of electrons from metals by ions, Phys. Rev. 96, 336 (1954).

[55] S. Kopelke, K. Gokhberg, V. Averbukh, F. Tarantelli, and L. S. Cederbaum, $A b$ initio interatomic decay widths of excited states by applying Stieltjes imaging to Lanczos pseudospectra, J. Chem. Phys. 134, 094107 (2011).

[56] See Supplemental Material at http://link.aps.org/supplemental/ 10.1103/PhysRevB.102.045408 for details regarding the samples used in our experiments, the charge exchange experiments using the electrostatic analyzer and the input parameters for the TDPot simulations.

[57] Note that we use the relation $R=\sqrt{\left(v_{\mathrm{p}} t\right)^{2}+b^{2}}$ to convert $\gamma(R(t))$ to $\gamma(t)$ with $v_{\mathrm{p}}$ as the initial projectile velocity and $b$ as the impact parameter.

[58] R. A. Wilhelm and W. Möller, Charge-state-dependent energy loss of slow ions. II. Statistical atom model, Phys. Rev. A 93, 052709 (2016).

[59] Y. Baskin and L. Meyer, Lattice constants of graphite at low temperatures, Phys. Rev. 100, 544 (1955).

[60] As a reference for nanopore sizes, we used the data from Ref. [9], which is for $180 \mathrm{keV}$ ions. In the present study, we use $87 \mathrm{keV}$ ions, which may lead to larger pores and therefore a larger damage area fraction.

[61] Charge exchange between highly charged ions and graphene was already investigated for samples produced by a polymerfree transfer method [17,52]. This is in contrast to the samples used in the present work, which where transferred onto the TEM grid using a polymer-based process and are cleaned post transfer in vacuo.

[62] W. Brandt, R. Laubert, M. Mourino, and A. Schwarzschild, Dynamic Screening of Projectile Charges in Solids Measured by Target X-Ray Emission, Phys. Rev. Lett. 31, 1095 (1973).

[63] M. Hattass, T. Schenkel, A. V. Hamza, A. V. Barnes, M. W. Newman, J. W. McDonald, T. R. Niedermayr, G. A.
Machicoane, and D. H. Schneider, Charge Equilibration Time of Slow, Highly Charged Ions in Solids, Phys. Rev. Lett. 82, 4795 (1999).

[64] N. Bohr, Velocity-range relation for fission fragments, Phys. Rev. 59, 270 (1941).

[65] S. Martin, R. Brédy, J. Bernard, J. Désesquelles, and L. Chen, Very Fast Hollow-Atom Decay Processes in $\mathrm{Xe}^{30+}-\mathrm{C}_{60}$ Collisions, Phys. Rev. Lett. 89, 183401 (2002).

[66] L. Folkerts, S. Schippers, D. M. Zehner, and F. W. Meyer, Time Scales for Charge Equilibration of $\mathrm{O}^{q+}(3 \leqslant q \leqslant 8)$ Ions During Surface-Channeling Interactions with $\mathrm{Au}(110)$, Phys. Rev. Lett. 74, 2204 (1995).

[67] A. Arnau, F. Aumayr, P. Echenique, M. Grether, W. Heiland, J. Limburg, R. Morgenstern, P. Roncin, S. Schippers, R. Schuch, N. Stolterfoht, P. Varga, T. Zouros, and H. P. Winter, Interaction of slow multicharged ions with solid surfaces, Surface Sci. Rep. 27, 113 (1997).

[68] D. Brandt, Resonant transfer and excitation in ion-atom collisions, Phys. Rev. A 27, 1314 (1983).

[69] J. A. Tanis, E. M. Bernstein, W. G. Graham, M. P. Stockli, M. Clark, R. H. McFarland, T. J. Morgan, K. H. Berkner, A. S. Schlachter, and J. W. Stearns, Resonant Electron Transfer and Excitation in Two-, Three-, and Four-Electron ${ }_{20} \mathrm{Ca}^{q+}$ and ${ }_{23} \mathrm{~V}^{q+}$ Ions Colliding with Helium, Phys. Rev. Lett. 53, 2551 (1984).

[70] Y. Guo, Z. Yang, Z. Song, Q. Xu, J. Chen, and B. Yang, L-shell X-ray production of molybdenum and niobium induced

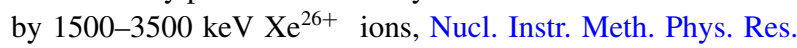
Sec. B 307, 7 (2013).

[71] H. Zhang, X. Chen, Z. Yang, J. Xu, Y. Cui, J. Shao, X. Zhang, Y. Zhao, Y. Zhang, and G. Xiao, Molybdenum $L$-shell x-ray production by $350-600 \mathrm{keV} \mathrm{Xe}^{q+}(q=25-30)$ ions, Nucl. Instr. Meth. Phys. Res. Sec. B 268, 1564 (2010).

[72] L. Zhaoyuan, Z. Hongqiang, C. Ximeng, Y. Zhihu, X. Jinzhang, C. Ying, X. Xu, S. Jianxiong, X. Guoqing, Z. Xiaoan, Z. Yongtao, and Z. Yanping, Interaction of highly charged $\mathrm{Xe}^{q+}$ ( $q=26,27,30)$ ions with molybdenum surface, Int. J. Mod. Phys. B 19, 2443 (2005). 